\title{
Assessment and management of alcohol dependence and withdrawal in the acute hospital: concise guidance
}

\author{
Stephen Stewart and Sharon Swain
}

\begin{abstract}
Alcohol dependence is common among patients attending acute hospitals. It can be the major reason for attendance or a significant cofactor. Assessment of these patients in the acute setting can be challenging owing to the multidisciplinary approach required. Doctors in acute hospitals are often inexperienced in managing dependence, a mental health problem. They might focus on the physical harms or the withdrawal, a consequence of the dependence. For this reason, assessment of dependence and prevention and management of acute alcohol withdrawal are often suboptimal. There is little existing guidance on how to manage this patient population, especially in non-specialist settings. With recently published National Institute for Health and Clinical Excellence (NICE) guidance on the management of dependence and withdrawal, now is the perfect time to produce concise guidelines in the hope that a more succinct suite of guidance can reach a larger audience.
\end{abstract}

KEY WORDS: alcohol dependence, alcohol withdrawal

\section{Introduction and aims}

When the National Institute for Health and Clinical Excellence (NICE) guidance for the management of alcohol-use disorders was divided into three separate components, ${ }^{1-3}$ this was in recognition of the multidisciplinary nature of these problems. Mental health and 'physical health' staff are both involved in caring for these patients, and hazardous and harmful drinking is a major public health issue.

We now have three comprehensive full guidelines ${ }^{1-3}$ and three quick reference guides. ${ }^{4-6}$ Continued effort is required to further integrate these guidelines and to increase their utility to the busy doctor at the coal face.

\footnotetext{
Stephen Stewart, ${ }^{1}$ consultant hepatologist and director for the Centre for Liver Disease; Sharon Swain, ${ }^{2}$ senior research fellow

${ }^{1}$ Mater Misericordiae University Hospital, Dublin; ${ }^{2}$ National Clinical Guidelines Centre

Adaptation from the original NICE guidelines commissioned by the Royal College of Physicians, as part of its Concise Guidance programme; on behalf of a multidisciplinary Guideline Development Group convened by the National Clinical Guidelines Centre and National Collaborating Centre for Mental Health; commissioned by the National Institute for Health and Clinical Excellence, and in association with the Clinical Effectiveness and Evaluation Unit of The Royal College of Physicians
}

This concise guideline is provided for doctors who encounter alcohol dependence and withdrawal in the course of their generic medical practice. It will specifically cover:

- the assessment of alcohol dependence

- the assessment of withdrawal, including the asssessment of need for admission to hospital

- the management of alcohol withdrawal, including delirium tremens and alcohol withdrawal seizures

- prophylaxis and treatment of Wernicke's encephalopathy.

\section{Methods}

The guidelines were developed in accordance with the relevant NICE guidelines manual. ${ }^{7,8}$

\section{Identification and assessment of harmful drinking and alcohol dependence}

The assessment of harmful drinking and alcohol dependence starts with the taking of an alcohol history. Recent and longterm alcohol consumption is often assessed in the emergency department or medical and/or surgical admission units with one or two questions. In some cases, this is adequate, but in many patients, more detailed assessment is required. This is particularly important in cases where alcohol is a contributing factor to the admission, but in any patient, there might be hazardous or harmful drinking that could be managed with brief intervention if brought to light.

Staff in acute settings need to be able to recognise the alcoholdependent patient. This requires vigilance and an index of suspicion and can be aided by standardised tools (see guidance in Fig 1 and Fig 2). Once these patients are recognised, they can then be channelled towards help for their addiction and monitored for signs and symptoms of withdrawal.

1 Use formal assessment tools to assess the nature and severity of alcohol misuse, including the:

- Alcohol Use Disorders Identification Test (AUDIT) for identification and as a routine outcome measure (Fig 1)

- Severity of Alcohol Dependence Questionnaire (SADQ) (Fig 2) ${ }^{13}$ or Leeds Dependency Questionnaire (LDQ) $)^{9}$ for severity of dependence

- Clinical Institute Withdrawal Assessment of Alcohol Scale, revised (CIWA-Ar) ${ }^{10}$ for severity of withdrawal

- Alcohol Problems Questionnaire (APQ) ${ }^{11}$ for the nature and extent of the problems arising from alcohol misuse. ${ }^{1}$ 
2 When assessing the severity of alcohol dependence and determining the need for assisted withdrawal, adjust the criteria for women, older people, children and young people, and people with established liver disease who might have problems with the metabolism of alcohol. ${ }^{1}$

\section{Assessment for the need to admit for medically assisted withdrawal}

Not all patients attending an acute setting with alcohol withdrawal need to be admitted to hospital. The decision around admission can be complex and the recommendations are to provide guidance rather than to act as strict criteria.
Many dependent patients manage their alcohol withdrawal symptoms every day with continued alcohol consumption and it is often appropriate to continue this until they can be assessed formally by addiction services to determine the best treatment for their alcohol dependence. There are situations in which this is inappropriate:

- severe, or impending severe, withdrawal is the main reason to admit

- delirium tremens and seizures are absolute criteria for admission

- autonomic overactivity and evidence of withdrawal with a high blood alcohol concentration would raise concerns about impending severe withdrawal

Fig 1. The Alcohol Use Disorders Identification Test (AUDIT) questionnaire. ${ }^{12}$ Scoring the questionnaire: scores for each question range from 0 to 4 , with the first response for each question (eg never) scoring 0 , the second (eg less than monthly) scoring 1 , the third (eg monthly) scoring 2 , the fourth (eg weekly) scoring 3 , and the last response (eg daily or almost daily) scoring 4 . For questions 9 and 10 , which only have three responses, the scoring is 0,2 and 4 . A score of 8 to 15 is associated with harmful or hazardous drinking. A score of 16 to 19 is harmful, mild or moderate dependence; $\geq 20$ is severe dependence. ${ }^{1}$ Copyright World Health Organisation 2001.

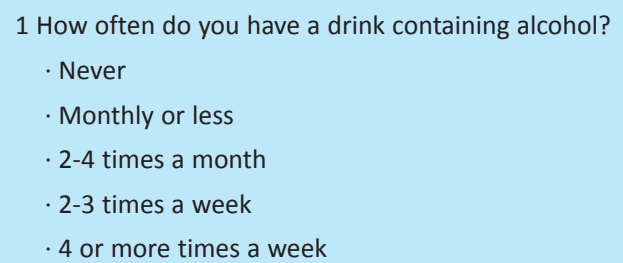

2 How many standard drinks containing alcohol do you have on a typical day when drinking?

$$
\begin{aligned}
& \cdot 1 \text { or } 2 \\
& \cdot 3 \text { or } 4 \\
& \cdot 5 \text { or } 6 \\
& \cdot 7 \text { to } 9 \\
& \cdot 10 \text { or more }
\end{aligned}
$$

3 How often do you have six or more drinks on one occasion?

$$
\text { - Never }
$$$$
\text { - Less than monthly }
$$

4 During the past year, how often have you found that you were not able to stop drinking once you had started?

$$
\begin{aligned}
& \text { - Never } \\
& \text { - Less than monthly } \\
& \text { - Monthly } \\
& \text { - Weekly } \\
& \text { - Daily or almost daily }
\end{aligned}
$$

5 During the past year, how often have you failed to do what was normally expected of you because of drinking?

$$
\begin{aligned}
& \text { - Never } \\
& \text { - Less than monthly } \\
& \text { - Monthly } \\
& \text { - Weekly } \\
& \text { - Daily or almost daily }
\end{aligned}
$$

6 During the past year, how often have you needed a drink in the morning to get yourself going after a heavy drinking session?

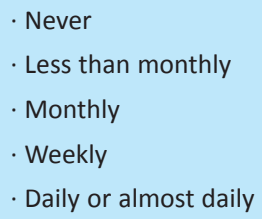

7 During the past year, how often have you had a feeling of guilt or remorse after drinking?

$$
\begin{aligned}
& \text { - Never } \\
& \text { - Less than monthly } \\
& \text { - Monthly } \\
& \text { - Weekly } \\
& \text { - Daily or almost daily }
\end{aligned}
$$

8 During the past year, have you been unable to remember what happened the night before because you had been drinking?

$$
\begin{aligned}
& \text { - Never } \\
& \text { - Less than monthly } \\
& \text { - Weekly } \\
& \text { - Daily or almost daily }
\end{aligned}
$$

9 Have you or someone else been injured as a result of your drinking?

$$
\text { - No }
$$

- Yes, but not in the past year

- Yes, during the past year

10 Has a relative or friend, doctor or other health worker been concerned about your drinking or suggested you cut down?

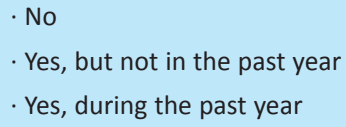


Fig 2. Severity of Alcohol Dependence Questionnaire (SADQ -C). ${ }^{13}$ Scoring the questionnaire: a score of 15 or less indicates mild dependence a score of 16-30 indicates moderate dependence; and a score of 31 or more indicates severe dependence. ${ }^{1}$

We would like to recall a recent month when you are drinking in a way which, for you, was fairly typical of a heavy drinking period. Please fill in the month and the year:

Month.

Year

We want to know more about your drinking during this time and how often you experienced certain feelings. Please put a tick to show how frequently each of the following statements applied to you during this typical period of drinking.

Score
0

Almost never
1

Sometimes
2

Often
3

Nearly always

1 I wake up feeling sweaty

$2 \mathrm{My}$ hand shaking first thing in the morning

3 My whole body shakes violently first thing in the morning, if I don't have a drink

4 I wake up absolutely drenched in sweat

5 I dread waking up in the morning

61 am frightened of meeting people first thing in the morning

7 I feel on the edge of despair when I wake up

8 I feel very frightened when I wake up

9 I like to have an alcoholic drink in the morning

10 I always gulp down my morning alcoholic drink as quickly as possible

11 I drink alcohol in the morning to get rid of the shakes

12 I have a very strong craving for an alcoholic drink when I wake up

13 I drink more than a quarter of a bottle of spirits in a day (or one bottle of wine, or seven beers)

14 I drink more than half a bottle of spirits per day (or two bottles of wine, or 15 beers)

15 I drink more than one bottle of spirits per day (or four bottles of wine, or 30 beers)

16 I drink more than two bottles of spirits per day (or eight bottles of wine, or 60 beers)

Imagine the following situation: You have been completely off drink for a few weeks and you then drink very heavily for two days. How would you feel the morning after those two days of drinking?

Not at all
Slightly
A lot

17 I would start to sweat

18 My hand would shake

19 My body would shake

20 I would be craving a drink 
- a history of seizures or delirium tremens would raise concerns but not mandate admission.

As with all patients, a general assessment of risk should be undertaken and certain vulnerable groups, such as the very young or the frail, would have a lower threshold for admission.

\section{Admission to hospital}

3 Make sure that assessment of risk is part of any assessment, that it informs the development of the overall care plan and that it covers risk to self (including unplanned withdrawal, suicidality and neglect) and risk to others. ${ }^{1}$

4 For people in acute alcohol withdrawal with, or who are assessed to be at high risk of developing, alcohol withdrawal seizures or delirium tremens, offer admission to hospital for medically assisted alcohol withdrawal. ${ }^{2}$

5 For young people under 16 years who are in acute alcohol withdrawal, offer admission to hospital for physical and psychosocial assessment, in addition to medically assisted alcohol withdrawal. ${ }^{2}$

6 For certain vulnerable people who are in acute alcohol withdrawal (eg those who are frail, have cognitive impairment or multiple comorbidities, lack social support, have learning difficulties or are 16 or 17 years old), consider a lower threshold for admission to hospital for medically assisted alcohol withdrawal. ${ }^{2}$

7 For people who are alcohol dependent but not admitted to hospital, offer advice to avoid a sudden reduction in alcohol intake and information about how to contact local alcohol support services. ${ }^{2}$

\section{The assessment and management of alcohol withdrawal}

Not all patients attending the acute setting with acute alcohol withdrawal will need pharmacotherapy. When patients do need medication, benzodiazepines are a good choice, with chlordiazepoxide and diazepam being the most commonly used. Pharmacotherapy should be delivered in a symptom-triggered manner, with the dose tailored to the patient's requirements. To individualise treatment, several factors have to be taken into account, including:

- the severity of dependence (discussed above)

- the severity of the withdrawal episode

- the patient's comorbidities.

For example, a young man with a high alcohol intake, a history of withdrawal seizures and normal liver function should be prescribed much higher doses of pharmacotherapy than a small older lady with cirrhosis who develops mild withdrawal on the background of a moderate alcohol intake. This might seem obvious, but many local hospital withdrawal treatment protocols will result in these patients getting the same dose of chlordiazepoxide.
There is evidence to support the use of tools to monitor the severity of withdrawal, such as the Clinical Institute Withdrawal Assessment - Alcohol, revised (CIWA-Ar) ${ }^{10}$ (Fig 3). This is not a substitute for the experience of a dedicated alcohol nurse specialist, but can be a useful adjunct to care for staff who might not have the same experience. In addition, these tools can be used repeatedly to monitor the course of the withdrawal and the results linked directly to the pharmacotherapy dose in a symptom-triggered regimen.

If alcohol withdrawal is managed well, delirium tremens and seizures should not be seen outside the setting of presentation to the acute hospital. However, these conditions are also seen if dependence is not recognised in a patient presenting with another condition or if withdrawal is suboptimally managed. The management then is to initiate pharmacotherapy or to increase the already initiated withdrawal regimen dose. In addition, lorazepam can be added as a short-acting benzodiazepine to halt or prevent further seizures or to control agitation in delirium. Haloperidol or olanzapine can be useful adjunctive treatments in delirium tremens. Anticonvulsants have no proven benefit in alcohol withdrawal seizures.

\section{Assessment and monitoring}

8 People in acute alcohol withdrawal should be assessed immediately on admission to hospital by a healthcare professional skilled in the management of alcohol withdrawal. ${ }^{2}$

9 Follow locally specified protocols to assess and monitor patients in acute alcohol withdrawal. Consider using a tool (eg the CIWA-Ar scale; Fig 3) as an adjunct to clinical judgement. ${ }^{2}$

\section{Management of withdrawal}

10 Offer drug treatment for the symptoms of acute alcohol withdrawal, as follows:

- consider offering a benzodiazepine or carbamazepine

- clomethiazole can be offered as an alternative to a benzodiazepine or carbamazepine. However, it should be used with caution, in inpatient settings only and according to the summary of product characteristics (SPC).

Follow a symptom-triggered regimen for the drug treatment of acute alcohol withdrawal in people who are:

- in hospital; or

- in other settings where $24 \mathrm{~h}$ assessment and monitoring are available.

11 In people with delirium tremens, offer oral lorazepam as first-line treatment. If symptoms persist or oral medication is declined, give parenteral lorazepam, haloperidol or olanzapine.

12 If delirium tremens develops in a person during treatment for acute alcohol withdrawal, review their withdrawal drug regimen. 
Fig 3. Clinical Institute Withdrawal Assessment of Alcohol Scale, revised (CIWA-Ar). ${ }^{10}$ Scoring ranges from 0 to 9 for minimal absent

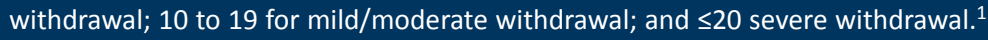

1 Nausea and vomiting: Do you feel sick to your stomach? Have you vomited?

0 no nausea and no vomiting

1 mild nausea with no vomiting

2

3

4 intermittent nausea with dry heaves

5

6

7 constant nausea, frequent dry heaves and vomiting

2 Tremor: Arms extended and fingers spread apart

0 no tremor

1 not visible, but can be felt fingertip to fingertip

2

3

4 moderate, with patient's arm extended

5

6

7 severe, even with arms not extended

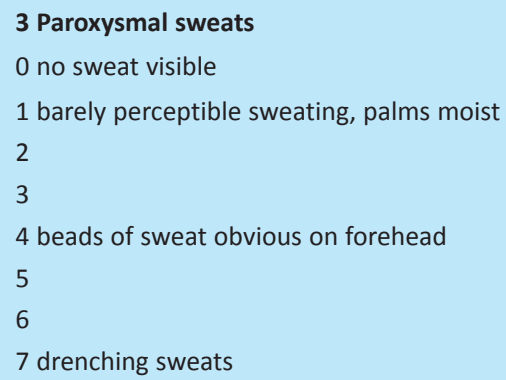

\section{Paroxysmal sweats}

0 no sweat visible

1 barely perceptible sweating, palms moist

2

3

4 beads of sweat obvious on forehead

5

6

7 drenching sweats

4 Anxiety: Do you feel nervous?

0 no anxiety, at ease

1 mildly anxious

2

3

4 moderately anxious or guarded, so anxiety is inferred

5

6

7 equivalent to acute panic states as seen in severe delirium or acute schizophrenic reactions

\section{Agitation}

0 normal activity

1 somewhat more than normal activitiy

2

3

4 moderately fidgety and restless

5

6

7 paces back and forth during most of the interview, or constantly thrashes about
6 Tactile disturbances: Have you any itching, pins and needles sensations, any burning, any numbness or do you feel bugs crawling on or under your skin?

0 none

1 very mild itching, pins and needles, burning or numbness

2 mild itching, pins and needles, burning or numbness

3 moderate itching, pins and needles, burning or numbness

4 moderately severe hallucinations

5 severe hallucinations

6 extremely severe hallucinations

7 continuous hallucinations

7 Auditory disturbances: Are you more aware of sounds around you? Are they harsh? Do they frighten you? Are you hearing anything that is disturbing you? Are you hearing things you know are not there?

0 not present

1 very mild harshness or ability to frighten

2 mild harshness or ability to frighten

3 moderate harshness or ability to frighten

4 moderately severe hallucinations

5 severe hallucinations

6 extremely severe hallucinations

7 continuous hallucinations

8 Visual disturbances: Does the light appear to be too bright? Is its colour different? Does it hurt your eyes? Are you seeing anything that is disturbing you? Are you seeing things you know are not there?

0 not present

1 very mildly sensitive

2 mild sensitivity

3 moderate sensitivity

4 moderately severe hallucinations

5 severe hallucinations

6 extreme severe hallucinations

7 continuous hallucinations

9 Headache, fullness in head: Does your head feel different? Does it feel like there is a band around your head? Do not rate for dizziness or lightheadedness. Otherwise rate severity.

0 not present

1 very mild

2 mild

3 moderate

4 moderately severe

5 severe

6 very severe

7 extremely severe

10 Orientation and clouding of sensorium: What day is this? Where are you? Who am I?

0 orientated and can do serial additions

1 cannot do serial additions or is uncertain about the date

2 disorientated for date by no more than 2 calender days

3 disorientated for date by more than 2 calender days

4 disorientated for place and/or person 
13 In people with alcohol withdrawal seizures, consider offering a quick-acting benzodiazepine (such as lorazepam) to reduce the likelihood of further seizures.

14 If alcohol withdrawal seizures develop in a person during treatment for acute alcohol withdrawal, review their withdrawal drug regimen.

15 Do not offer phenytoin to treat alcohol withdrawal seizures.

\section{The prophylaxis and management of Wernicke's encephalopathy}

It is essential to consider the diagnosis of Wernicke's encephalopathy in any confused dependent patient attending the acute hospital. The classic triad of ophthalmoplegia, ataxia and confusion is rarely seen and a high index of suspicion must be maintained along with a low threshold for treatment. The diagnosis of Wernicke's encephalopathy can be particularly challenging in patients who have another cause for their confusion, such as intoxication or hepatic encephalopathy. If there is any suspicion of Wernicke's, these patients should be treated with parenteral thiamine.

Prophylaxis is equally important. Decompensated liver disease, malnutrition or acute withdrawal mandates the use of prophylactic thiamine in the harmful or dependent drinker. Although this can be given orally in the community, it is recommended that, if a harmful or dependent drinker has either decompensated liver disease or evidence of malnutrition and attends the emergency department or is admitted to hospital, the opportunity to give at least one prophylactic dose of thiamine intravenously is taken.

\section{Wernicke's encephalopathy}

16 Offer thiamine to people at high risk of developing, or with suspected, Wernicke's encephalopathy. Thiamine should be given in doses toward the upper end of the British National Formulary range (200 mg/day in divided doses). It should be given orally or parenterally, as described in the recommendations below. Offer prophylactic oral thiamine to harmful or dependent drinkers:

- if they are malnourished or at risk of malnourishment; or

- if they have decompensated liver disease; or

- if they are in acute withdrawal; or

- before and during a planned medically assisted alcohol withdrawal.

17 Offer prophylactic parenteral thiamine followed by oral thiamine to harmful or dependent drinkers:

- if they are malnourished or at risk of malnourishment; or

- if they have decompensated liver disease; and in addition

- they attend an emergency department; or

- are admitted to hospital with an acute illness or injury.

18 Offer parenteral thiamine to people with suspected

Wernicke's encephalopathy. Maintain a high level of suspicion for the possibility of Wernicke's encephalopathy, particularly if the person is intoxicated. Parenteral treatment should be given for a minimum of five days, unless Wernicke's encephalopathy is excluded. Oral thiamine treatment should follow parenteral therapy.

\section{References}

1 National Institute for Health and Clinical Excellence. Alcohol-use disorders: diagnosis, assessment and management of harmful drinking and alcohol dependence (CG115). http://guidance.nice.org.uk/CG115/ Guidance/pdf/English [Accessed 27 March 2012].

2 National Institute for Health and Clinical Excellence. Alcohol-use disorders: diagnosis and clinical management of alcohol-related physical complications (CG100). http://guidance.nice.org.uk/CG100/Guidance/ pdf/English [Accessed 27 March 2012].

3 National Institute for Health and Clinical Excellence. Alcohol-use disorders: preventing harmful drinking (PH24). http://guidance.nice.org. uk/PH24/Guidance/pdf/English [Accessed 27 March 2012].

4 National Institute for Health and Clinical Excellence. Quick reference guide. Alcohol-use disorders: Diagnosis, assessment and management of harmful drinking and alcohol dependence (CG115). London: NICE, 2011b; http://guidance.nice.org.uk/CG115/QuickRefGuide/pdf/English [Accessed 27 March 2012].

5 National Institute for Health and Clinical Excellence. Quick reference guide. Alcohol-use disorders: Diagnosis and clinical management of alcohol-related physical complications. London: NICE, 2010. http:// guidance.nice.org.uk/CG100/QuickRefGuide/pdf/English [Accessed 27 March 2012].

6 National Institute for Health and Clinical Excellence. Quick reference guide. Alcohol-use disorders: Preventing harmful drinking (PH24). London: Nice, 2010. http://guidance.nice.org.uk/PH24/ QuickRefGuide/pdf/English [Accessed 27 March 2012].

7 National Institute for Health and Clinical Excellence. The guidelines manual 2009. www.nice.org.uk/aboutnice/howwework/developingniceclinicalguidelines/clinicalguidelinedevelopmentmethods/ GuidelinesManual2009.jsp [Accessed 27 March 2012].

8 National Institute for Health and Clinical Excellence. The guidelines manual 2007. London: NICE 2007. www.nice.org.uk/media/FA1/59/ GuidelinesManualChapters2007.pdf [Accessed 14 May 2012].

9 Raistrick D, Bradshaw J, Tober G et al. Development of the Leeds Dependence Questionnaire (LDQ): a questionnaire to measure alcohol and opiate dependence in the context of a treatment evaluation package. Addiction 1994;89:563-72.

10 Sullivan JT, Sykora K, Schneiderman J et al. Assessment of alcohol withdrawal: the revised clinical institute withdrawal assessment for alcohol scale (CIWA-Ar). Br J Addict 1989;84:1353-7.

11 Drummond, C. The relationship between alcohol dependence and alcohol related problems in a clinical population. Br J Addict 1990:85;357-66.

12 Barbor TF, Higgins-Biddle JC, Saunders J. B, Montera MG. AUDIT: the alcohol use disorders identification test. Guidelines for use in Primary Care, 2nd edn. Geneva: World Health Organisation, 2001.

13 Stockwell T, Hodgson R, Edwards G et al. The development of a questionnaire to measure severity of alcohol dependence. Br J Addict Alcohol Other Drugs 1979:74;78-87.

Address for correspondence: Dr Stephen Stewart, Centre for Liver Disease, 55 Eccles Street, Dublin 7, Ireland.

Email: sstewart@mater.ie 